For reprint orders, please contact: reprints@futuremedicine.com

\title{
Design and functionality of a smart fentanyl iontophoretic transdermal system for the treatment of moderate-to-severe postoperative pain
}

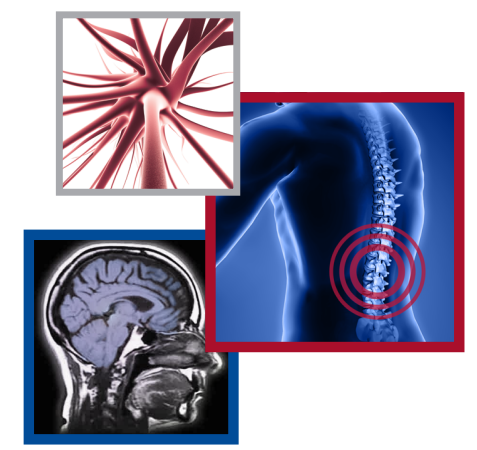

Nitin Joshi' ${ }^{1}$ John Lemke1 \& Hassan Danesi*,

\section{Practice points}

Passive versus active transdermal delivery of drugs for acute pain management

- Passive transdermal delivery is controlled by drug diffusion.

- Active transdermal delivery, such as iontophoresis, involves an active delivery mechanism allowing rapid delivery of a drug.

lontophoresis

- Iontophoresis allows for noninvasive delivery of medication across intact skin.

- The rate of fentanyl delivery is proportional to the magnitude of applied current and independent of the concentration of fentanyl in the reservoir.

Fentanyl-Separated System with Enhanced Controller

- The fentanyl Separated System with Enhanced Controller consists of a drug unit and controller that are snapped together immediately prior to the application to the patient; this new system eliminates the issue of corrosion that was originally seen with the first-generation system.

- The controller was designed and developed as a medical device.

- The drug unit contains fentanyl.

- The ionic current results in the delivery of fentanyl ions across the skin and into systemic circulation.

System self-testing

- Self-testing occurs when the system is assembled and repeated throughout the use period.

Reliability

- The design incorporates features to prevent damage to the dose control system and is fail-safe, with redundant protective measures that detect faults and shut the system down safely.

- No evidence of corrosion or any unsafe condition has been observed in over 10,000 systems tested during stability testing.

How this technology relates to the pharmacokinetics of fentanyl ITS

- Bioequivalence has been demonstrated between the first- and second-generation fentanyl ITS systems. 
Fentanyl iontophoretic transdermal system (ITS) is a patient-controlled analgesia system used for the management of acute postoperative pain. The first-generation fentanyl ITS was an integrated one-piece system; however, corrosion that could limit reliability was detected in a small number of systems. A second-generation fentanyl ITS was designed to separate the hydrogels in the Drug Unit from the electronic circuit of the Controller during manufacture and storage, removing the primary cause of corrosion and thereby improving reliability. No evidence of corrosion has been observed in over 10,000 systems tested in real-time aging studies for the second generation fentanyl ITS. The second generation fentanyl ITS design features combine to ensure safe operation of the system with high reliability.

First draft submitted: 26 November 2015; Accepted for publication: 24 December 2015; Published online: 25 January 2016

\section{KEYWORDS}

- design • fentanyl • IONSYS

- iontophoretic transdermal system • patient-controlled analgesia
Fentanyl iontophoretic transdermal system (ITS; IONSYS ${ }^{\circledR}$, The Medicines Company, NJ, USA) is a needle-free, patient-controlled analgesia system that is used for the short-term management of acute postoperative pain in adult patients requiring opioid analgesia during hospitalization [1-7]. The first-generation system was approved by the US FDA on 22 May 2006; however, it was never marketed due to reliability concerns that could lead to a selfinitiating system. After conducting a detailed failure investigation, the company concluded that the co-packaging of the electronics within the same primary packaging as the hydrogels exposed the electronics to extreme humidity that could damage the electronics and potentially result in self-initiating systems. The first-generation fentanyl ITS was an integrated system. The second-generation of the fentanyl ITS is designated as the Separated System with Enhanced Controller (SSEC) and separates the hydrogels in the drug unit from the electronic circuit of the controller during manufacture and storage (Figure 1A). The healthcare professional assembles the system by snapping the drug unit and controller together immediately prior to application to the patient (Figure 1B). The fentanyl ITS User Interface is detailed in Figure 1C. The active ingredient in fentanyl ITS is fentanyl (10.8 mg fentanyl hydrochloride equivalent to $9.7 \mathrm{mg}$ fentanyl). The secondgeneration fentanyl ITS is approved for use by the FDA as of 30 April 2015 for the shortterm management of acute postoperative pain in adult patients requiring opioid analgesia in the hospital. On 19 November 2015, fentanyl ITS received EC approval for the management of acute moderate-to-severe, postoperative pain in adult patients for use in the hospital.
Passive versus active transdermal delivery of drugs for acute pain management

Passive transdermal drug delivery refers to one in which there is no mediation beyond application of the patch to the skin. The amount of drug delivered from a passive patch is controlled by concentration gradient driven drug diffusion through the skin. The drug is at a high concentration in the patch to provide a concentration gradient. Passive transdermal delivery with opioids is frequently utilized in chronic pain management; however, it is not indicated [8] in acute pain because products such as the transdermal fentanyl patch have a slow onset of action (plateauing in $\sim 15 \mathrm{~h}$ ) [9,10]. The passive patch also leaves a depot in the tissue resulting in slow offset of action - a significant amount of drug continues to be absorbed in the blood stream for several hours after the patch is removed [11]. Due to these pharmacokinetic features, passive transdermal patches of potent drugs such as fentanyl are not indicated for opioid naïve patients and counter indicated for the management of acute pain. In comparison to this passive process, iontophoresis is an active delivery mechanism - mediation beyond application of the system to skin is required in order to deliver the drug. The technique of iontophoresis allows for rapid transdermal delivery of a drug (such as fentanyl) compared with the slower process of passive transdermal absorption. The amount of passive fentanyl delivery from an iontophoretic system is negligible; thereby enabling drug delivery strictly in an on-demand or active mode. The ability to accurately control dosing is the biggest advantage of iontophoretic systems over passive patches. Additionally, there is no depot effect with an iontophoretic system. As a result, there is no significant drug delivery after the system is 


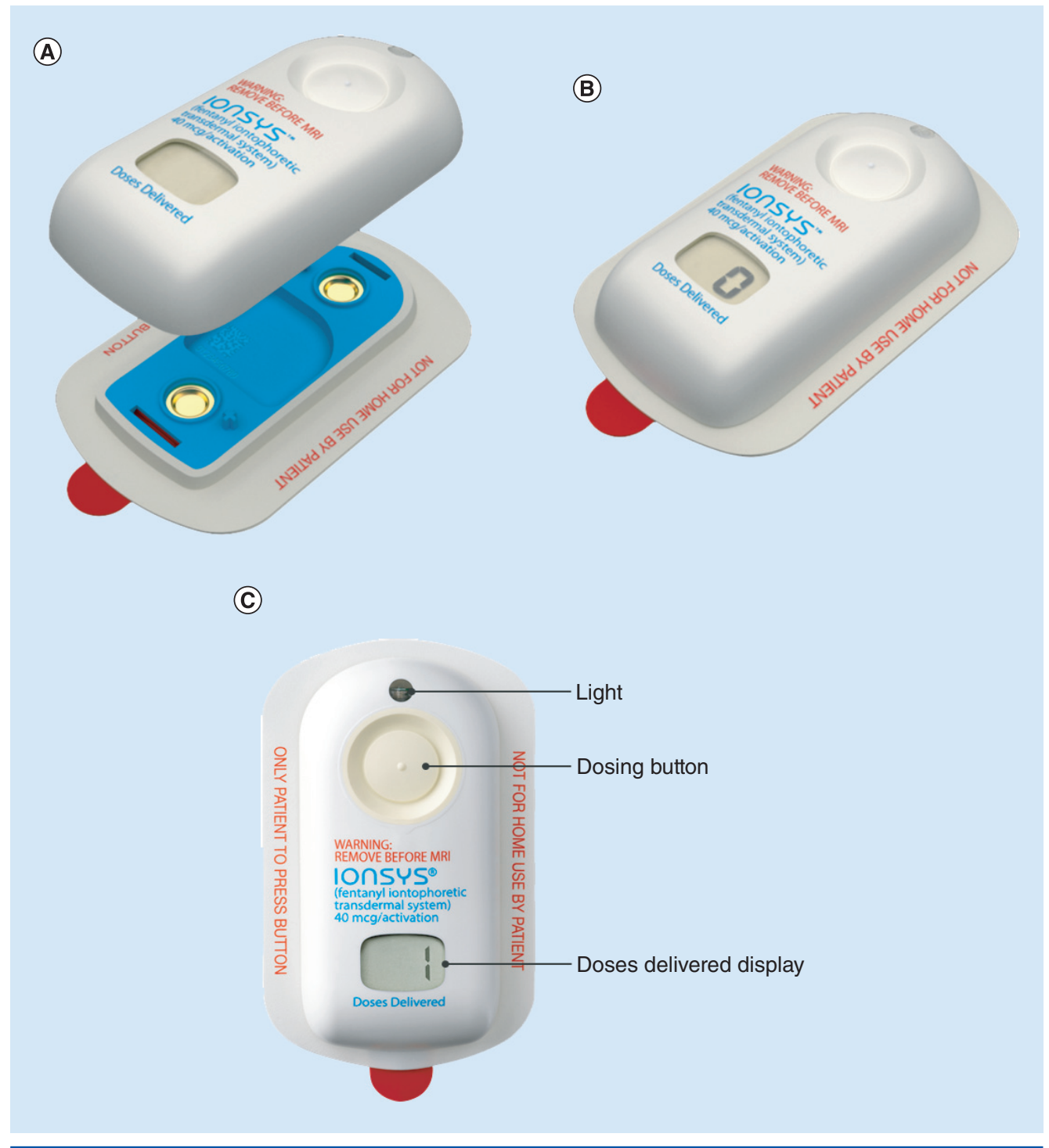

Figure 1. Illustration of the fentanyl Separated System with Enhanced Controller and drug unit. (A) Controller and drug unit prior to assembly. (B) Assembled fentanyl SSEC system. (C) Fentanyl SSEC user interface.

SSEC: Separated system with enhanced controller.

Reproduced with permission from The Medicines Company (NJ, USA).

removed from the patient's skin. These benefits make an iontophoretic system ideally suited to acute pain management.

\section{Iontophoresis}

Transdermal drug delivery using iontophoresis was originally described over 40 years ago [12] Iontophoresis allows for noninvasive delivery of therapeutic substances across intact skin. An iontophoretic drug delivery system consists of a power supply connected to a pair of electrodes in contact with ionically conductive reservoirs that, in turn, are in contact with the skin.
The rate of transdermal delivery of fentanyl ion, $\mathrm{R}_{\mathrm{d}}$, from the anode hydrogel formulation is governed by the following expression derived from Faraday's Laws of Electrolysis:

$R_{d}=\frac{t_{d} I M_{d}}{z_{d} F}$

where, $t_{d}$ is the fentanyl transport number (fraction of charge that is carried by the fentanyl ion), I is the current, $M_{d}$ is molecular weight of fentanyl, $z_{d}$ is the valence of the fentanyl ion $(+1)$ and $\mathrm{F}$ is Faraday's constant (electric charge per mole of electrons). Therefore, the rate of fentanyl 
delivery is directly proportional to the magnitude of the applied current. The rate can be linearly adjusted by adjusting the current. The total amount of fentanyl delivered to the patient depends on the rate of fentanyl delivery and the duration of delivery. In effect, the total fentanyl dose is directly proportional to the charge, which is the product of current and time.

It is important to note that the rate of fentanyl delivery is independent of the concentration of fentanyl in the reservoir. This is unlike passive patches that are driven purely by concentration gradient of the drug and, therefore, depend on a saturated drug reservoir. It requires the drug to be in solution (dictated by solubility) and in an ionic state (dictated by $\mathrm{pH}$ ).

The factors that determine transdermal iontophoretic delivery are analogous to delivery of a drug dissolved at a fixed concentration in an aqueous reservoir using an intravenous (IV) pump. The rate of drug delivery with an IV pump is proportional to the magnitude of the solution flow rate (analogous to flow of electric current). The amount of drug delivered to the patient is determined by the duration of the flow. The product of the flow rate and the duration of flow determine the total volume of solution (analogous to charge) delivered to the patient. The amount of fentanyl delivered to the patient by transdermal iontophoresis is determined by the amount of charge delivered across the skin, analogous to the volume of solution at a fixed concentration delivered to the patient with an IV pump.

\section{Fentanyl Separated System with Enhanced} Controller design

Fentanyl SSEC consists of a drug unit and controller that are snapped together immediately prior to application to the patient (Figure 1A \& B) . The controller has been designed and developed as a medical device. The drug unit contains the pharmaceutical component, which is essentially unchanged from the previously approved integrated system.

The controller is comprised of three elements: controller top, printed circuit board assembly and the controller bottom (Figure 2). The printed circuit board assembly is protected by the ultrasonically welded plastic controller top and bottom housings. The printed circuit board assembly consists of a push button switch to initiate dosing, an integrated circuit to monitor the switch and control the dosing current and time, a resistor used by the integrated circuit to monitor the dosing current, a coin cell battery to supply system power, and an inductor, diode and capacitor comprising a voltage boost circuit. The integrated circuit controls all operations of the SSEC and was developed specifically for this application. The integrated circuit contains a microcontroller and a mask ROM (read-only memory) with the controller software. A digital to analog converter generates the dose current. A 12-bit analog to digital converter monitors system parameters. A hardware reset function monitors battery voltage, clock frequency, redundant voltage references and a watchdog timer that monitors software operation. If any of these values are out of specification, the system shuts down in a safe mode. The integrated circuit also contains drivers for the liquid crystal display (LCD), bi-color light-emitting diode (LED), audio transducer and interfaces for external memory and manufacturing test functions. The battery is a standard CR2032 coin cell installed on the printed circuit board assembly after the surface mount technology parts are assembled on the board. It is a primary cell battery, meaning it is for single use and not rechargeable. The battery is enclosed within the controller's top and bottom housings and is not user-accessible. The CR2032 battery is specified for nominal $3 \mathrm{~V}$ output, $225 \mathrm{mAh}$ capacity, at continuous load of $0.2 \mathrm{~mA}$ with a specified operating temperature range of $-30^{\circ} \mathrm{C}$ to $+60^{\circ} \mathrm{C}$. To prevent corrosion during storage, power from the battery is not applied to the circuit during manufacturing or storage, but is automatically applied when the system is snapped together by the healthcare professional at time of use. Two power-on switches are connected in series with one another and are normally in an open state. They close upon proper assembly of the controller and the drug unit, connecting the battery to the circuit. Upon receiving a legitimate dose request, the controller applies a constant current of $0.170 \mathrm{~mA}$ for $10 \mathrm{~min}$ for the drug delivery function and automatically performs self-tests periodically throughout the entire 24-h use period during all modes of operation.

The drug unit consists of four main elements: interface module, bottom housing assembly, skin adhesive and release liner (Figure 2). All of the components of the SSEC that are in contact with the skin are contained within the drug unit. The bottom housing assembly is a thermoformed part with two gel cavities, two electrodes (anode and cathode) and two hydrogels (anode and cathode). The bottom housing 

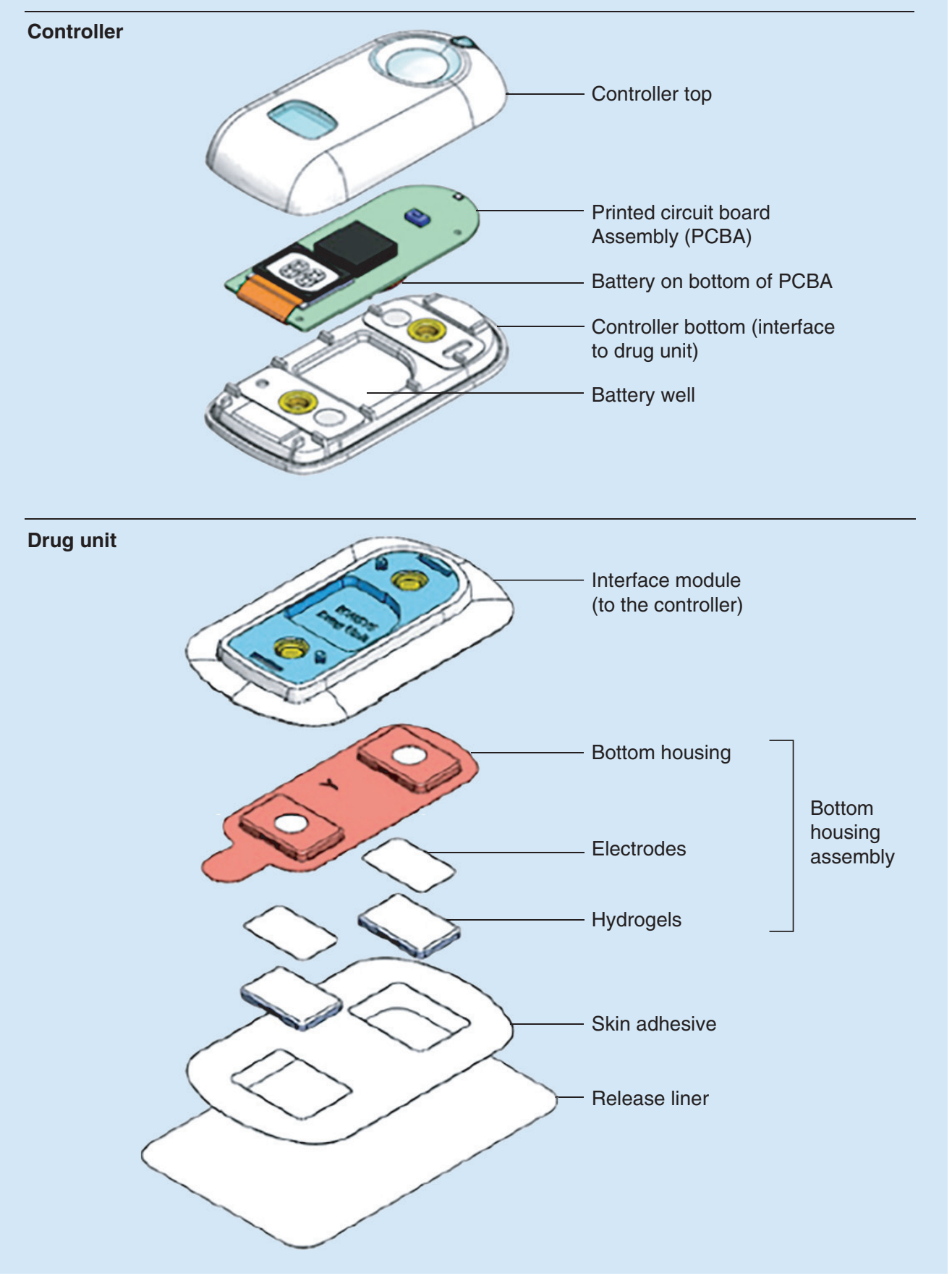

Figure 2. Fentanyl iontophoretic transdermal system Separated System with Enhanced Controller detailed schematic.

Reproduced with permission from The Medicines Company (NJ, USA).

assembly has the same formulation and uses the same materials as the first-generation system. The interface module is a plastic injection molded part that is utilized to connect the drug unit to the controller. In addition to providing electrical and mechanical connection between the drug unit and the controller, it has flexible elastomeric wings along the sides to help attach the system to the patient's skin. The peripheral skin adhesive covers the bottom housing assembly and the interface module without covering the hydrogels. A release liner is applied over 


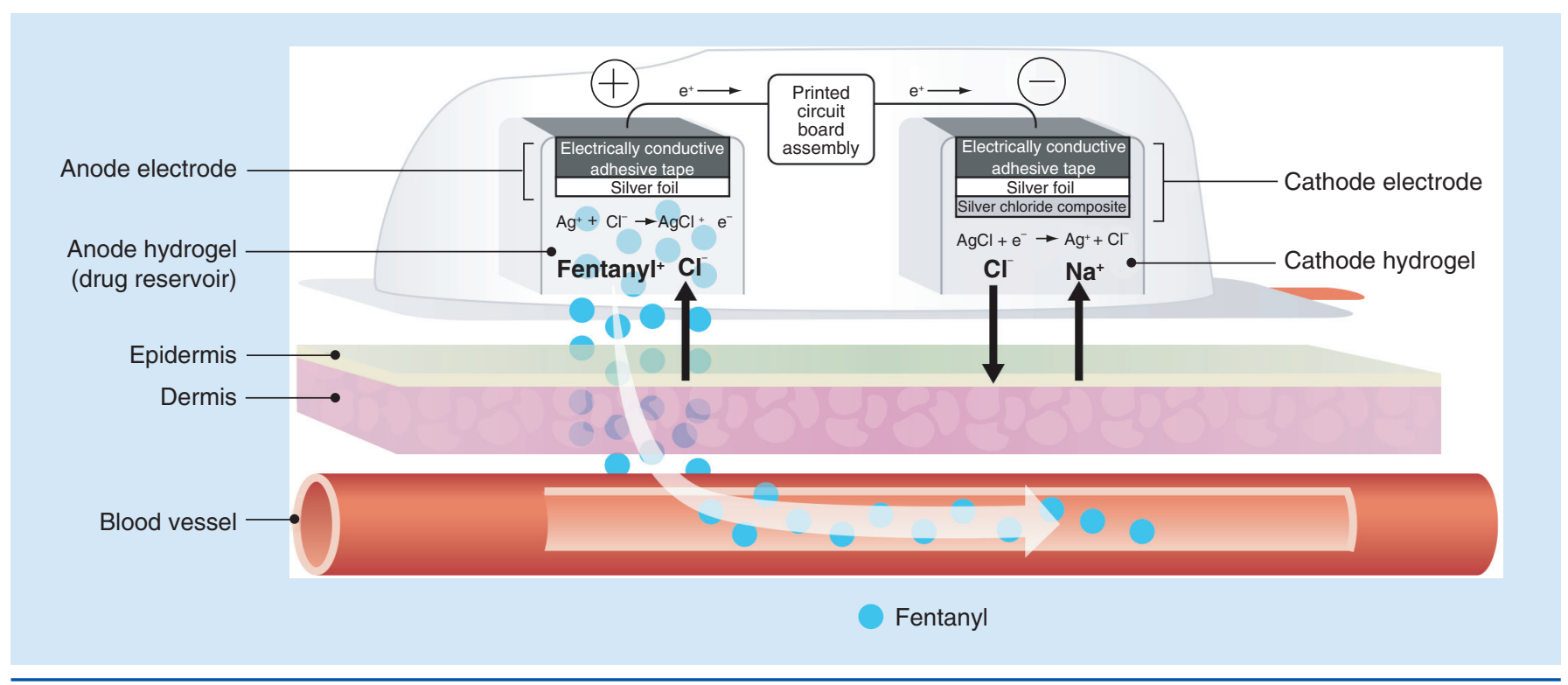

Figure 3. Iontophoretic delivery of fentanyl.

Reproduced with permission from The Medicines Company (NJ, USA).

the skin adhesive prior to sealing the drug unit inside a foil pouch. The release liner is removed by the healthcare professional before applying the SSEC to the patient. The SSEC uses the same skin adhesive, release liner and foil pouch materials as the first-generation system.

The drug unit contains anode and cathode electrodes and hydrogels that convert the electrical current from the controller into the flux of fentanyl ions through the skin. The formulation of the electrodes and hydrogels provides the elements for the electrochemical reactions that enable the precise delivery of fentanyl from the SSEC. The hydrogels contact the skin, and a small ionic current of $0.170 \mathrm{~mA}$ is applied when the system is activated by the patient. The ionic current results in the delivery of fentanyl ions across the skin and into systemic circulation. A schematic of the iontophoretic process for delivering fentanyl ions to the patient is shown in Figure 3. The anode electrode and hydrogel deliver the positively-charged fentanyl ion into the body. The cathode electrode and hydrogel close the electrical circuit. Each hydrogel is placed in contact with the patient's skin and contains either fentanyl (for the anode electrode assembly) or a pharmacologically inactive electrolyte (for the cathode electrode assembly). During dosing, the silver anode is oxidized, forming silver cations. The silver cations react with chloride anions in the anode hydrogel to form silver chloride on the surface of the silver anode. Simultaneously, silver chloride in the cathode is reduced to form silver metal and chloride anions. To maintain charge neutrality within the anode and cathode hydrogels, cations and anions are exchanged with the body, resulting in the delivery of fentanyl cations into the skin and extraction of chloride and sodium ions from the body.

The current density distribution over the surface of the anode and cathode hydrogels was measured to confirm that current is uniformly dispersed over the skin contact area with no areas of high current density. The anode and cathode hydrogels are $2.0 \mathrm{~cm} \times 1.3 \mathrm{~cm}$ in dimensions. The gel resistance at 15 points distributed over the surface of each hydrogel was measured for newly manufactured systems, systems aged for 6 months, unused systems and systems that had been used for delivering 40 doses. The resistance varied from 13 to $32 \mathrm{k} \Omega$ for the anode gel and 17 to $30 \mathrm{k} \Omega$ for the cathode gel. These translate to a maximum variation of $\pm 20 \%$ in current density across the surface of either gel. The nominal output current required for drug delivery is $0.170 \mathrm{~mA}$, and the total dose charge is held constant by the controller. Therefore, the observed variation in current density is not likely to have any significant impact on either drug delivery or the incidence of application site reactions.

The drug hydrogel of the fentanyl ITS contains $9.7 \mathrm{mg}$ of fentanyl, which is less than the amount in a high-dose passive fentanyl patch. After all 80 doses are used up, $6.7 \mathrm{mg}$ of fentanyl 
remains in the system. This amount is required to ensure that 80 doses of $40 \mu \mathrm{g}$ each are reliably delivered by the system. Therefore, it is important to adhere to proper disposal procedures that are described in the prescribing information.

- Assembly of the drug unit to the controller \& system self-testing

When assembled to the drug unit, the controller automatically confirms that the hardware conditions (correct battery and reference voltages and timer signal) necessary for the micro-controller to operate properly are present before booting up the software. If any of these conditions are not met, the system would remain off.

After successful power-up, the controller ensures that the system operates within specifications at all times during use by a series of self-tests. On assembly, self-tests conducted prior to use by the patient verify that the calibration values stored in the integrated circuit are valid, confirm that the voltage control circuits are operating within specification, confirm that internal timers are operating accurately and confirm that the output current is off. Self-tests are repeated throughout the use period. Additionally, during dosing, the output current is measured every second to confirm that it is within specifications. To initiate a dose, the patient has to press and release the dose button twice within $3 \mathrm{~s}$. A valid dose request is defined by these two consecutive voltage transitions within a specified period of time. The double-button press requirement prevents accidental dose initiation. This feature is the same as that in the original ITS. The SSEC design includes a self-test that confirms that the speed of signal transitions within the dose initiation circuit is consistent with a human button press - if the transitions are too fast it ignores these as noise and does not initiate a dose. In addition, prior to initiating a dose, the SSEC design verifies that voltage levels within the dose initiation circuit are within specification. In the event that any of the tests described above fail or detect a potentially unsafe condition, the controller shuts the system down safely and permanently.

\section{- Reliability}

The SSEC met all design requirements during Design Verification and Validation testing, confirming that the SSEC dose control system provides the same fentanyl delivery as the firstgeneration system. The SSEC design incorporates features to prevent damage to the dose control system and is fail-safe, with redundant protective measures that detect faults and shut the system down safely.

Highlights of the second-generation design include separation of the electronics and hydrogels during storage; removal of voltage bias from the electronic circuit during storage; sealing the electronics within the controller housing; system self-testing procedures conducted immediately after assembly of the controller to the drug unit; switch-signal verification after every double

Table 1. Patents for the second-generation system.

\begin{tabular}{|c|c|c|}
\hline Patent date & Patent number & Brief description \\
\hline 30 October 2012 & US 8,301,238 B2 & $\begin{array}{l}\text { Describes a two-part electrotransport therapeutic agent delivery } \\
\text { device, such as an iontophoresis device. The two parts are } \\
\text { provided separately and assembled just prior to use }\end{array}$ \\
\hline 23 April 2013 & US 8,428,708 B1 & $\begin{array}{l}\text { Describes the methods, devices and systems for monitoring } \\
\text { and controlling electrotransport drug delivery devices to detect } \\
\text { and/or prevent delivery of drug by the device when it is in an off } \\
\text { mode or state }\end{array}$ \\
\hline 23 April 2013 & US 8,428,709 B1 & $\begin{array}{l}\text { Describes an electrotransport drug delivery system including } \\
\text { constant current delivery systems having a feedback current } \\
\text { and/or voltage control module that is isolated from the patient } \\
\text { contacts (e.g., anodes and cathodes). The feedback current and/ } \\
\text { or voltage control measurements are performed at the transistor } \\
\text { rather than at the patient contact (e.g., cathode) }\end{array}$ \\
\hline 18 February 2014 & US D699,855 S & $\begin{array}{l}\text { Describes the ornamental design for an iontophoretic drug } \\
\text { delivery device }\end{array}$ \\
\hline 15 July 2015 & US 8,781,571 B2 & $\begin{array}{l}\text { Describes the switch-operated therapeutic agent delivery device, } \\
\text { which can provide single or multiple doses of therapeutic agents } \\
\text { to a patient by activating a switch }\end{array}$ \\
\hline
\end{tabular}


button press to confirm the signal profile is consistent with patient activation; periodic self-test to confirm integrity of switch and dosing circuits throughout the use period; and, component and manufacturing test requirements and specifications. These features combine to ensure the safe operation with high reliability required by the SSEC user.

By separating the electronics and hydrogels, the SSEC design eliminates the corrosion that was observed with the first-generation system. No evidence of corrosion or any unsafe condition has been observed in over 10,000 systems tested during stability testing for the SSEC.

\section{- Instructions for use}

Fentanyl ITS is indicated for use only in a hospital and is removed by the healthcare professional before the patient leaves the hospital. Hospitals have to be certified as per a productspecific Risk Evaluation and Mitigation Strategy (REMS) program before they can receive and use Fentanyl ITS. The REMS certification ensures that the hospital has adequate systems to use and dispose the product. It also ensures that staff will be trained on proper use before using the product. Fentanyl ITS is a prefilled, preprogrammed, single-use system for up to 80 discreet doses of $10 \mathrm{~min}$ each over a maximum duration of $24 \mathrm{~h}$. It delivers a fixed dose of $40 \mu \mathrm{g}$ /activation. After the 10-min dose is complete, the system goes back to the ready state and the patient can request another dose. Up to $6 \times 40 \mu \mathrm{g}$ doses are available per hour. Each system is intended to be used for up to $24 \mathrm{~h}$ or 80 doses, whichever comes first; the system then automatically disables drug delivery after which time the device is to be disposed. If any attempt is made to separate the two parts of the Fentanyl ITS, it automatically shuts down and cannot be restarted or reused.

\section{- Patents}

The fentanyl SSEC design is innovative, and during the development of the second-generation system there were a series of patents issued that cover this updated device (Table 1). The first one was issued on 30 October 2012 (Patent No. US $8,301,238$ B2) and covers the two-part electrotransport device. In this patent, a switch-operated therapeutic agent delivery device is described. The second patent was issued on 23 April 2013 (Patent No. 8,428,708 B1) and covers the selftest for the analgesic product. The third patent was issued on 23 April 2013 (Patent No. US $8,428,709 \mathrm{~B} 1$ ) and covers the current control for electrotransport drug delivery. The fourth patent was issued on 18 February 2014 (Patent No. US D699,855S) and covers the iontophoretic drug delivery system. The fifth patent was issued on 15 July 2014 (Patent No. US 8,781,571 B2) and covers the switch validation circuit and method.

\section{- How this technology relates to pharmacokinetics of fentanyl ITS}

Throughout the development program of the SSEC, the main design principle has been to replicate all the key aspects from the first-generation that controlled fentanyl delivery, while enhancing product reliability. Bioequivalence has been demonstrated between the first-generation and the second-generation fentanyl ITS systems in a pharmacokinetic study that compared dose absorbed between the two systems [13]. The bioequivalence results indicate that the secondgeneration system will provide the same clinical efficacy as the first-generation ITS.

\section{Acknowledgements}

The authors wish to thank S Grundy of SD Scientific, Inc. for her technical writing contribution to the manuscript, funded by The Medicines Company.

Financial \& competing interests disclosure

The authors are employees of The Medicines Company. The authors have no other relevant affiliations or financial involvement with any organization or entity with a financial interest in or financial conflict with the subject matter or materials discussed in the manuscript apart from those disclosed.

Writing assistance was utilized in the production of this manuscript.

\section{References}

Papers of special note have been highlighted as:

- of interest; $\bullet$ of considerable interest

1 Chelly JE, Grass J, Houseman TW, Minkowitz H, Pue A. The safety and efficacy of a fentanyl patient-controlled transdermal system for acute postoperative analgesia: a multicenter, placebocontrolled trial. Anesth. Analg. 98(2), 427-433 (2004).

- Describes the safety and efficiacy of fentanyl iontophoretic transdermal system (ITS).

2 Grond S, Hall J, Spacek A, Hoppenbrouwers M, Richarz U, Bonnet F. Iontophoretic transdermal system using fentanyl compared with patient-controlled intravenous analgesia using morphine for postoperative pain management. Br. J. Anaesth. 98(6), 806-815 (2007).

- Describes the safety and efficiacy of fentanyl ITS. 
3 Hartrick CT, Bourne MH, Gargiulo K, Damaraju CV, Vallow S, Hewitt DJ. Fentanyl iontophoretic transdermal system for acute-pain management after orthopedic surgery: a comparative study with morphine intravenous patient-controlled analgesia. Reg. Anesth. Pain Med.31(6), 546-554 (2006).

-• Describes the safety and efficiacy of fentanyl ITS.

4 Minkowitz HS, Rathmell JP, Vallow S, Gargiulo K, Damaraju CV, Hewitt DJ. Efficacy and safety of the fentanyl iontophoretic transdermal system (ITS) and intravenous patient-controlled analgesia (IV PCA) with morphine for pain management following abdominal or pelvic surgery. Pain Med. 8(8), 657-668 (2007).

-• Describes the safety and efficiacy of fentanyl ITS.

5 Viscusi ER, Reynolds L, Chung F, Atkinson LE, Khanna S. Patient-controlled transdermal fentanyl hydrochloride vs intravenous morphine pump for postoperative pain: a randomized controlled trial. JAMA 291(11), 1333-1341 (2004).

6 Viscusi ER, Reynolds L, Tait S, Melson T, Atkinson LE. An iontophoretic fentanyl patient-activated analgesic delivery system for postoperative pain: a double-blind, placebocontrolled trial. Anesth. Analg. 102(1), 188-194 (2006).

$7 \quad$ IONSYS $^{\circledR}$ (fentanyl iontophoretic transdermal system), prescribing information. The Medicines Company, USA (2015). www.accessdata.fda.gov

-. Provides the approved labeling for the product, including a summary of product features, clinical results, side effect profile and proper use and disposal instructions.

8 Duragesic (Fentanyl Transdermal System), prescribing information.

www.duragesic.com

- Compares passive and active fentanyl delivery, and the approved label for Duragesic provides a good description of a passive fentanyl patch.

9 Grond S, Radbruch L, Lehmann KA. Clinical pharmacokinetics of transdermal opioids: focus on transdermal fentanyl. Clin. Pharmacokinet. 38(1), 59-89 (2000).

- Provides a review and summary of transdermal delivery of fentanyl.
10 Holley FO, Van Steennis C. Postoperative analgesia with fentanyl: pharmacokinetics and pharmacodynamics of constant-rate i.v. and transdermal delivery. Br. J. Anaesth. 60(6), 608-613 (1988).

11 Lehmann KA, Zech D. Transdermal fentanyl: clinical pharmacology. J. Pain Symptom Manage. 7(Suppl. 3), S8-S16 (1992).

12 Echols DF, Norris CH, Tabb HG. Anesthesia of the ear by iontophoresis of lidocaine. Arch. Otolaryngol. 101(7), 418-421 (1975).

13 Phipps JB, Joshi N, Regal KA, Li J, Sinatra RS. Pharmacokinetic characteristics of fentanyl iontophoretic trandermal system over a range of applied current. Expert Opin. Drug Metab. Toxicol. 11(4), 481-489 (2015).

-• Describes results from a human pharmacokinetic study that compares the old and new design of fentanyl ITS. The results summarized in this paper demonstrate that the new design provides similar drug delivery and absorption as the old system. 